\title{
Daily real-time monitoring of the PV thin film modules for analysis and system modeling
}

\begin{abstract}
The power output of a Photovoltaic system is mainly influenced by the weather conditions. Semitransparent [CdTe] Thin-Film PV module is one of the promising green energy sources that need to be more investigated in terms of efficiency and modeling. This paper presents real-time daily measurements for such modules that serve the system design, prediction, and modeling. Single and multi-layer configurations categories have been used as a platform to test the PV system at various temperature and irradiance ranges. Experimental measurements were conducted at UPM University, Serdang, Malaysia, where a 6 different modules are tested according to their interconnection and installation. A wireless data acquisition card, of one second sampling interval, has been utilized to acquire the weather and electrical parameters (module temperature, solar irradiance, Voltage, and Current). The result of the harvested daily energy shows a value around (2.51) as a maximum efficiency in a cloudy day conditions and it is influenced by the transparency factor when configured in a multi-layer. It is found that the modules' performance is similar to that of a Silicon-based solar panels.
\end{abstract}

Keyword: PV thin-film; Photovoltaic systems; System efficiency; Real-time monitoring; PV modeling 